\title{
Ordinary Thinking about Time
}

\author{
John Campbell, Berkeley
}

\section{Why do it the ordinary way?}

I will describe two non-standard ways of thinking about time. The first is ubiquitous in animal cognition. I will call it 'phase time'. Suppose for example you consider a hibernating animal. This animal might have representation of the various seasons of the year, and modulate its actions dependent on the season. But it need have no distinction between the winter of one year and the winter of another; it thinks of time only in terms of repeatable phases.

The second non-standard way of thinking about time has been ascribed to children at an early stage in development. I will call it 'script time'. A 'script' or 'schema' is representation of the structure of a repeated type of event, such as going to a restaurant, attending a lecture or visiting the doctor. You know what types of event happen in what order. And in script time, you identify temporal locations with respect to events in the script.

Within each of these ways of thinking of time there is a recognizable "earlier than" relation. In phase time, the relation is not transitive. In script time, the "earlier than" relation only holds between times within the same script. Both these ways of thinking of time contrast with our ordinary conception.

It is not immediately obvious just how to draw the contrast. You might say that we ordinarily think of time as linear, so that "earlier than" is transitive and connected. That is certainly how our use of calendars and clocks suggests we think. But does our ordinary way of thinking of time in autobiographical memory and in planning for the future demand the full strength of transitivity and connectedness?

Why do we use our ordinary way of thinking of time, rather than the nonstandard ways? You might say, "This is simply what we do", and argue that no explanation can be given. I shall argue, though, that we can explain why we think of time as we do by looking at the way we make sense of the transmission of causal influence from place to place by concrete objects. I begin by setting out the two non-standard ways of thinking of time in more detail. 


\section{Phase time}

Consider an animal using a circadian clock. The clock dictates when the animal sleeps and wakes, when it feels hungry, and so on, for a number of routine procedures through which it goes every day. So far, though, we might have here only what we might call biological time. It is one thing for an animal to have biological mechanisms that are time-sensitive, another for it to have temporal cognition. A sun-flower seed might germinate at a particular time of year; that does not of itself show that the seed is representing time. What does it take for an organism to have not merely biological, but cognitive time? A natural answer is that it has to do with whether the animal can be viewed as performing computations over temporal representations (Gallistel 1990). The sunflower seed does not perform computations relating to the time of year. On the other hand a foraging bird, for example, determining its rate of return for the time spent in a particular field, might be engaging in quite complex calculations concerning time. So if we consider an animal with a circadian clock, we can say that we have properly cognitive time if we have an ability to use its temporal knowledge in finding, for example, the optimum plan for the day, the optimum order in which to perform various tasks and how long to spend on them.

The agent knows what phase the day is currently at: whether it is early, late or mid-morning, for example. And the agent may have discovered and stored information about what typically happens at various particular phases of the day - that breakfast is served at 10.00am on Forel's balcony, for example. And the agent may put this stored information to use in guiding action, as honeybees used to gather at Forel's balcony at breakfast time (Gallistel 1990, 243). So the agent arrives for food at the right time of day, and leaves shortly afterwards. Notice, though, that the agent so far has only the conception of time as (repeatable) phase. The agent does not draw, and makes no use of, the distinction between the morning of one day and the morning of another.

The domain of times over which the agent's temporal representations are defined is oriented: adjacent times are ordered by "earlier than". Early morning precedes mid-morning. But the domain is cyclically ordered, somewhat as places on the equator are cyclically ordered by "to the east of". The "earlier than" relation here is not transitive, just as "to the east of" is not transitive.

To be able to use its circadian clock, there must be some sense in which the agent can use temporal indexicals: terms like "now" or "in a little while". 
The agent has to be able to represent, "it's mid-morning now", or "in a little while it will be mid-morning". The agent can do this by using indexicals which are governed by rules whose formal statement is exactly like that of the rules governing our ordinary temporal indexicals. Just as we have a term, "now", whose reference is fixed by the rule I will call 'Linear':

Linear: Any token of "now" refers to the time at which it was produced,

so the agent will use a term "now" governed by the rule I will call 'Phase':

Phase: Any token of "now" refers to the time at which it was produced.

The similarity between the two rules, Linear and Phase, is I hope evident. The difference between them is in the domain of times over which they are defined. Linear is defined over the everyday domain of unrepeatable moments, linearly ordered by "earlier than". Phase, on the other hand, is defined over the domain of phases, such as "early morning" "mid-morning" and so on, which are cyclically ordered by "earlier than". Despite this difference, a system of indexicals governed by rules such as Phase, will serve the animal for practical purposes-planning, action and so on-somewhat as our ordinary indexicals, defined over a domain of linearly organized times, serve us. There surely is a sense in which indexicals are essential (Perry 1979), but indexicals governed by rules defined over domains of phase times will do; it is not essential that we have indexicals governed by rules defined over domains of linearly organized times.

There seems, indeed, to be no reason why we could not have, within phase time, temporal operators for which we need Reichenbach's distinction between time of utterance, reference time and event time (Reichenbach 1947). It is just that again, the domain of times over which the notions are defined will be a domain of phases. And the "earlier than" relation will be a relation defined over times as phases. So we could have representations such as "when $X$ happens, $Y$ will have happened already". Here the opening clause defines the reference time, with respect to which the event $Y$ is then temporally located. So it is not just that we can have rudimentary indexicals in phase time; we can have a relatively sophisticated set of tenses and temporal operators.

One way to bring out the difference between an agent operating with phase times and an agent operating with linear time is to remark that there 
is a sense in which the agent operating with phase times does not have an authentic past tense. The agent can indeed have an operator, "in the past, ", governed by a rule:

"In the past,__ " is true if at some time earlier than the time of utterance,

But there is a sense in which this is not an authentic past tense, as I will now explain. There are some types of event that I am powerless to affect: the weather, the tides, the rise of hip-hop, and so on. I am no more able to affect tomorrow's weather than I am to affect yesterday's weather. When you and I speak of events in the past, however, there is a sense in which their temporal location alone renders them insusceptible to being affected by us. Past events may be events of types that, in general, I am able to affect. What I have for breakfast is, within limits, something over which I have a lot of control each day. But I cannot now affect what I had for breakfast yesterday. This is not a matter of the event being of a type that I am in general unable to affect. It has to do entirely with the event being past; or, if you prefer, with the event being earlier than the time at which I am attempting to act.

No such conception of the past is available to an agent with only the conception of time as phase. Suppose as before that I am able to affect what is for breakfast. Whether there is marmalade for breakfast at $10.00 \mathrm{am}$ is then something that I can affect. Just after breakfast on one morning, I can think, "breakfast is just over". Early the next morning I will be able to think, "Breakfast is just about to come up". But the only conception I have of there being marmalade for breakfast is the conception of a state of affairs I am currently able to change. I can think of there being marmalade for breakfast at $10.00 \mathrm{am}$; but that is the state of affairs I am currently able to change. I can affect whether there is, in general, marmalade for breakfast at $10.00 \mathrm{am}$. I can't now affect whether there was marmalade for breakfast yesterday at $10.00 \mathrm{am}$. But that conception of a particular, unrepeatable time, yesterday at $10.00 \mathrm{am}$, is just what is not available to an agent who has only the conception of time as phase.

You might argue that the agent may have the episodic memory of yesterday's marmalade at breakfast, a memory of that particular event, which is indeed unchangeable by the agent now. My present point, however, is that the agent does not have the conception of the temporal location of the event as that which makes the event incapable of being affected by the agent now; 
and that point remains even if the agent does have, say, a memory-image of yesterday's event. However, once this point is grasped, it seems questionable whether the agent could be said to have a truly episodic memory of that individual event, since the agent does not have the conception of the event as having a particular temporal location. It is not just that the agent does not know exactly when the past event occurred. Rather, the agent does not even have the conception of there being a particular time at which the past event occurred. In what sense then does the agent have the conception of this as a particular event at all?

So much, for the moment, for the conception of time as phase. As I said, this conception is ubiquitous in animal cognition. It is usually said that only humans have the conception of time as linear. But animal timing is commonplace. And one element in animal's representation of time is the representation of phase time. This is what I have been trying to characterize.

\section{Script time}

Young children seem able to form and retain information about the temporal structure of observed sequences. For example, there are cases of deferred imitation, in which children can repeat observed action sequences after a delay. McCormack and Hoerl (1999) make the point that the information retained here may be, as they say, generic, rather than relating to the temporal relations between the specific events observed. The information is generic in that it is "information about a temporal structure numerically different event sequences may have and which, therefore, does not distinguish between one event sequence and another." (McCormack and Hoerl $1999,158)$. So there is a sense in which the child has learned from observation of the past events, but what the child has may be generic information, rather than episodic memory of the particular events initially observed, and the temporal relations among them.

McCormack and Hoerl describe this type of generic memory as involving the construction of scripts, in something like the sense of Schank and Abelson (1977); cf also Nelson (1986). A script represents what usually happens in a situation in which there is a well-established pattern to what happens, such as going to a seminar, visiting a doctor, or eating in a restaurant. Having a script is having a representation that can, as it were, talk you through the situation. McCormack and Hoerl propose that scripts function as 'temporal frameworks' for young children. They provide ways of representing the 
temporal locations of novel events. You may recognize a sequence of events as falling under a script even if it contains some unexpected elements; the script itself provides a framework of times with respect to which the temporal locations of the unexpected elements can be plotted.

As McCormack and Hoerl stress, there is a sense in which the framework provided by a script is non-perspectival: scripts do not of themselves locate events with respect to one's present temporal position. However, here as in the case of phase time, perspectival representations will be needed if the temporal framework is to be put to use in practice. How are we to find the temporal token-reflexives that we will need to express a perspectival representation of time? We might use terms such as "now", governed by the rule:

Script: Any token of "now" refers to the time at which it was produced.

The domain of times over which this rule is defined will not, of course, be times drawn from our ordinary range of linearly organised times; they will themselves be times defined in terms of the temporal framework provided by the script. Within each script times are temporally related; but we cannot express temporal relations between times identified in different scripts.

In virtue of what do we have our ordinary understanding of temporal indexicals, rather than a more primitive conception? McCormack and Hoerl suggest that the key difference here, the marker of a mature grasp of temporal indexicals, is a certain capacity for temporal decentring, "where decentring means being able to conceive of other times as affording alternative temporal perspectives." (p. 171).

They first describe a simple type of temporal decentring, which is not enough for the ordinary conception of time. This kind of decentring is put to work in understanding any use of the present tense to refer to a time other than the time at which the understanding takes place. This kind of decentring is used by any child who manages to follow a narrative beginning with the words, "Once ..." or "One day ...", followed by quotations of present-tense utterances. For example, if I say, "Once, as I stood in line, someone said to me, 'you're standing on my foot'", anyone following the narrative has to interpret the quoted present-tense utterance "You're standing on my foot" as relating to a time other than the time at which they are hearing the narrative.

McCormack and Hoerl suggest that this simple decentring can be described as form of perspective-switching, in that the child who can do this 
may not so much as be able to make sense of the question what temporal relations hold between the various perspectives themselves. At this stage all the child has is the ability to switch from one script-time to another scripttime, and may have no ability to inquire into the temporal relations between the various script times. They say:

We wish to suggest that temporal decentring, if it is to be more than the perspective-switching described above, must similarly involve a conception of temporal perspectives as perspectives onto the same temporal reality and an understanding of the systematic relations that obtain between different temporal perspectives in virtue of this fact. Understanding that there can be multiple temporal perspectives onto the same event, for example, involves being able to reason that a current ongoing event will be in the past from the perspective of subsequent days and was in the future from the perspective of previous days. (p. 174)

And it is in virtue of our possession of this richer capacity for temporal decentring, they suggest, that we can be said to have the mature conception of time. In the terms I suggested, their proposal is that it is this richer capacity for temporal decentring that constitutes operating with our ordinary understanding of temporal indexicals, rather than the more primitive indexicals I have been describing.

It seems to me that there is a role for temporal decentring in an analysis of our ordinary understanding of time, but that this approach does not identify that role correctly. For example, consider an agent who does not have our ordinary conception of time, but does have the conception of phase time, as well as various scripts. It would be possible to have the conception of a single network of phases onto which all scripts could be plotted. For instance, suppose our agent has the conception of the various phases of the day: morning, afternoon, evening and night, and perhaps divides them into early morning, mid-morning, late morning and so on. Such an agent might be unable to distinguish the times of one day from the times of another day; this agent can only talk and think about those phases. In effect, this agent has a single script for the day, onto which all events are plotted. So this agent would not be operating with a range of more or less specialized scripts, just a single general-purpose script for a typical day. Such an agent would be capable of decentring in the simple sense: this agent could decentre to consider what happens in the early morning or in the mid-afternoon, 
for example, and this agent could interpret present-tense utterances considered as made at those times. But our agent could also consider the temporal relations between those various phase-times; our agent could reflect on the relation between early morning and mid-afternoon, for instance, reflecting on the intervening phases of mid- and late-morning, and early afternoon. So this subject would meet the McCormack/Hoerl characterisation of rich decentring, without yet having our ordinary understanding of temporal indexicals. It therefore does not seem that it could be the capacity for rich decentring that explains our ordinary understanding of temporal indexicals.

\section{Causation and physical objects}

It is natural to suspect that what is missing here is an understanding of the relation between grasp of our ordinary conception of time and a grasp of causal concepts. I want to approach this question in terms of the interventionist approach to causation developed by a number of authors (Pearl 2000, Spirtes, Glymour and Scheines 1993, Woodward 2003). The idea here is that for a variable $X$ to be a cause of $Y$ is for intervening on $X$ to be a way of intervening on $Y$. We can explain this more explicitly by introducing the notion of an 'intervention' variable (Woodward and Hitchcock 2003). For I to be an intervention variable for $X$ with respect to $Y$, it must be that:

$I$ causally affects $X$.

$I$ does not causally affect $Y$ otherwise than by affecting $X$.

$I$ is not correlated with any variable $Z$ that is correlated with $Y$ through a route that excludes $\mathrm{X}$.

$I$ suspends any other variable from affecting the value of $X$.

Suppose all these conditions are met by the intervention variable. Suppose that $X$ remains correlated with $Y$ under interventions on $X$. The correlation then cannot be due to some common cause of $X$ and $Y$; the conditions above exclude that possibility. So it can only mean that there is a causal connection between $X$ and $Y$. The interventionist says: for $X$ to be a cause of $Y$ is for $X$ and $Y$ to be correlated under interventions on $X$. Or to put it another way, for $X$ to be a cause of $Y$ is for intervening on $X$ to be a way of intervening on $Y$.

This characterization of causation itself makes free use of the notion of cause. That does not mean that the characterization is trivial. It does not 
merely explain " $X$ causes $Y$ " in terms of the idea that $X$ causes $Y$; on the contrary, it does not appeal at all to the idea of a causal connection specifically between $X$ and $Y$. The fact that the characterization itself uses the notion of cause does, however, mean that there is a question whether it makes explicit all the ideas that are required for an ordinary understanding of causation. In particular, it leaves it open whether an understanding of temporal concepts is required for a grasp of causation; and if so, what kind of temporal concepts. I want to end with some remarks on this.

The interventionist account is a counterfactual account; if it were a reductionist counterfactual account in the style of Lewis, it is hard to see how it could avoid using temporal concepts at some point. If we explain " $X$ causes $Y$ " by saying that there are counterfactual circumstances in which a difference in $X$ would make a difference to $Y$, we have to say just which kind of circumstances we have in mind. For a Lewis-style account, an appeal to time will be needed to specify just what we have to hold constant and what we are varying. So we might say that we will hold constant everything about the way the world is up until the time at which there is a difference in $X$. And here we really will need the ordinary notion of time, not merely phase time or script time. Because the interventionist account claims the right to make free use of the notion of cause in characterizing cause, however, the interventionist has another way of saying what is to be held constant and what varied when the value of $X$ changes. That is exactly what is achieved by the four conditions I quoted above. And there is here no explicit appeal to temporal notions. In explaining what it is for an ordinary subject to have grasped these four conditions, it will be natural at some point to appeal to the subject's own capacity for agency, a tendency in some situations to regard one's own actions as interventions (Gopnik et. al. 2004). But this kind of approach needs to appeal only to the subject's sensitivity to the causal relations in which her own actions stand to other phenomena. It does not, on the face of it anyhow, have to appeal to the subject's grasp of time at all.

Suppose, though, that we look at the details of our ordinary understanding of how causal influence is transmitted from place to place by physical objects. It is really basic to our understanding of ourselves as spatially located that what happens at one place can have repercussions for what happens at other places. Places are not causally insulated from one another. But neither are places promiscuously related by causation. It is not as if what happens at any one place affects any arbitrary range of other places. So how is causal influence transmitted from place to place? If you think about the 
surface of a pond, you might think of waves fanning out from one place to another set of locations, and causal influence being transmitted in that way. But there is another way of thinking that we often use. We think that causal influence is transmitted from place to place by the movement of objects. I light an oil heater in the garden and then find it's cold indoors, so I bring the heater in. Because of the movement of the heater, my intervention, my lighting of the gas at one place, makes a difference to the temperature at another place. We would be baffled by the idea that my lighting the heater outside could have made a difference to the temperature of the room without the heater having been moved, unless of course we appeal to some other story about heat waves or the motion of molecules. The movement of the physical thing, the oil heater, explains how it is that my intervention outside is making a difference to the temperature inside. This implies:

(a) there is more to the movement of a physical object than there being correlations under interventions between what goes on at one place and what goes on at another, and

(b) this further sameness of physical object at one place with physical object at another place is what explains the existence of those correlations between what goes on at one place and what goes on at the other, under interventions at one of the places.

We could sum all this up by saying that there is more to our ordinary concept of causation than the interventionist account allows. In addition to the fact that an intervention on one variable makes a difference to the value of another, we think there is, in many cases, a mechanism by which this happens. Ordinary physical objects are just the very simplest examples of such mechanisms. An intervention at one location can make a difference at another location; and movement of the object from one location to another is the mechanism by which this transmission of influence from one location to another is achieved.

It is this dimension of our ordinary understanding of causation that demands the ordinary conception of time. Suppose we have an intelligent agent operating with phase time only, making no distinction between one morning and another. An object may be observed one morning to be $G$ when the previous morning it had been observed to be F. And our agent can recognize a counterfactual dependence of the $\mathrm{G}$-ness on the F-ness of that object. If there had been an intervention on the F-ness of the object, it 
would not be G. As I said, formulating this kind of counterfactual does not seem to require use of temporal ideas. What our agent cannot do is identify the ground of the counterfactual dependence. For the ground of the counterfactual is the persisting object itself, transmitting causal influence from its earlier place to its present place. Recognizing that temporal relation demands that our agent go beyond the confines of phase time, towards something more like our ordinary thinking about time.

Consider now an intelligent agent using only script time. In running a number of scripts over a period of time, the agent may find that one and the same object is encountered in the course of many different scripts: a parent, for example, may figure in trips to the doctor, restaurant and so on. And the behavior of that thing in the running of one script may counterfactually depend on what happened in the running of another script. Merely stating the counterfactual dependence, if this is done in interventionist terms, does not require the use of temporal concepts. But recognizing the ground of the counterfactual connection, in the persistence of the object that figures in the running of one script to its figuring in the running of another script, requires recognizing temporal relations that go across scripts. So we have to move away from the series of temporal islands provided by script time, to a more connected conception of time.

This is not to say that recognizing the role of persisting physical objects as the mechanisms that transmit causal influence from place to place will demand the full strength of the conception of time as linear. When the introduction of a calendar and clock system makes linearity explicit, this is a bold, simple stroke that clarifies our thinking about time. But the pressure to recognize transitivity and connectedness is already there when we consider the temporal structure that has to be recognized by a self-conscious agent. In general, we believe in the transitivity of the connectedness of the self, in this sense: if an intervention on the self's F-ness would have made a difference to its G-ness, and an intervention on the self's G-ness would have made a difference to its $\mathrm{H}$-ness, then an intervention on the self's Fness would have made a difference to its $\mathrm{H}$-ness. And we think of the self as a concrete object that transmits causal influence from every place remembered to every place remembered or anticipated. This is a special case, albeit a particularly striking one, of the general point that it is our conception of the causal roles of concrete objects that explains the structure of our ordinary understanding of time. 


\section{Acknowledgement}

This paper benefited from a lively discussion at the Wittgenstein Symposium in 2005; thanks in particular to Jeremy Butterfield and Robin LePoidevin. I am also indebted to Naomi Eilan, Christoph Hoerl and Teresa McCormack for many discussions of these topics.

\section{References}

Gallistel, C. R 1990 The Organization of Learning. Cambridge, MA: MIT Press.

Gopnik, Alison, Clark Glymour, David Sobel, Laura Schulz, Tamar Kushnir and David Danks 2004 "A Theory of Causal Learning in Children: Causal Maps and Bayes Nets", Psychological Review 111, 1-31.

Lewis, David 1973 “Causation”, Journal of Philosophy, 70, 556-67.

McCormack, Teresa and Christoph Hoerl 1999 "Memory and Temporal Perspective: the Role of Temporal Frameworks in Memory Development", Developmental Review, 19, 154-182.

Nelson, Katherine 1986 Event Knowledge: Structure and Function in Development. Hillsdale, N. J.: L. Erlbaum Associates.

Perry, John 1993 "The Essential Indexical". In: The Essential Indexical and Other Essays. New York: Oxford University Press.

Reichenbach, Hans 1947 Elements of Symbolic Logic. New York: Macmillan.

Pearl, Judea 2000 Causation. Cambridge: Cambridge University Press.

Schank, Roger C., and Robert P. Abelson 1977 Scripts, Plans, Goals, and Understanding: An Inquiry into Human Knowledge Structures. Hillsdale, NJ: Lawrence Erlbaum.

Spirtes, Peter, Clark Glymour and Richard Scheines 1993 Causation, Prediction and Search. New York: Springer-Verlag.

Woodward, James 2003 Making Things Happen: A Theory of Causal Explanation. Oxford: Oxford University Press.

Woodward, James and Christopher Hitchcock 2003 "Explanatory Generalizations, Part 1: A Counterfactual Account", Nous 37, 1-24. 